American Journal of Applied Sciences 6 (2): 317-327, 2009

ISSN 1546-9239

(C) 2009 Science Publications

\title{
Design of New Load Shedding Special Protection Schemes for a Double Area Power System
}

\author{
H. Seyedi, M. Sanaye-Pasand \\ Control and Intelligent Processing Center of Excellence \\ School of Electrical and Computer Engineering, \\ University of Tehran, PO Box 14395-515, Tehran, Iran
}

\begin{abstract}
In this paper several Under Frequency Load Shedding (UFLS) schemes are designed as special protection schemes to preserve the integrity of islands, formed following the outage of tie lines connecting two areas in a double area power system. For the purpose of analysis, the Iranian interconnected grid is simulated in a suitable power system analysis software. The grid is connected to the Khorasan province network, the greatest province of the country, through a double circuit tie line. Following the outage of tie lines in a double area power system the area with great import of power may face large deficiency of energy and consequently blackout may occur in the island. The purpose of this research work is to propose a special protection scheme, being capable of preserving the balance of generation and consumption in order to prevent major outage. Special types of adaptive UFLS scheme are proposed in this work.
\end{abstract}

Keywords: Power System Protection, Special Protection Schemes, Load Shedding

\section{INTRODUCTION}

Major power system breakdowns have been occurring historically in interconnected electric grids. In recent years economical restrictions in the installation of new power system components especially transmission facilities, which may be a consequence of power system deregulation, has considerably increased the frequency and intensity of power system blackouts ${ }^{[1,2]}$.

The following factors play important roles in the recently occurred blackouts:

- Incorrect operation of protective systems, especially distance relays ${ }^{[3]}$.

- Voltage instability ${ }^{[4-8]}$.

- Inefficient design or lack of Under Frequency Load Shedding system ${ }^{[4-9]}$.

- Lack of under voltage load shedding system $^{[4-8]}$. - Inefficient vegetation management strategies

In this paper the third item, i.e. UFLS system, is considered and studied. UFLS scheme could be categorized in a class of protective systems known as system protection schemes, special protection schemes or wide area protection schemes.

UFLS scheme is one of the most generally used system protection schemes in power systems. Preliminary work on this issue started in 1950's. However it was accepted by most utilities after the 1965 northeastern blackout ${ }^{[12]}$. UFLS systems are conventionally designed to prevent excessive frequency decline, following the outage of some generating units, by preserving the balance of generation and consumption. Whenever frequency of the system falls below a predetermined value, some part of system load will be dropped after a time delay.

In most of the recently occurred power system blackouts two or more islands emerge as a consequence of the disturbance ${ }^{[4-9]}$. Any island which is capable of preserving the balance of generation and consumption may survive, otherwise it will cascade to blackout.

UFLS scheme, as a type of special protection scheme, may help the island preserve balance and prevent cascading outage. Conventional UFLS schemes are designed to protect the system against normal power deficiencies as in the case of one or two generating unit outage or sudden increase of load. Conventional UFLS scheme reaction is usually insufficient in the case of cascading outages, since in this case islands usually face excessive power deficiency. Therefore, in the case of large imbalances between generation and consumption UFLS scheme can not generally perform appropriately since it has not been designed specifically for these types of events. Adaptive UFLS schemes might be able to prevent complete system blackouts in the case of large imbalances.

In conventional under frequency protection design the

Corresponding Author:

H. Seyedi, School of Electrical and Computer Engineering, University of Tehran, PO Box 14395515, Tehran, Iran, Tel: +98-2166911444, Fax: +98-2188633029 
only measured parameter of the system, involved in decision making, is frequency. Whenever frequency of the system falls below a predefined threshold, a portion of system load is disconnected in a few steps. Although this type of under frequency load shedding is simple and easy to implement, especially with electromechanical relays, it suffers from some disadvantages. The most important disadvantage of this scheme is its lack of adaptability. In other words, regardless of the severity of disturbance, settings of the under frequency load shedding schemes are constant. This lack of adaptability may result in either overshedding or undershedding in different situations.

Several conventional and adaptive UFLS schemes have been proposed in the literature ${ }^{[13-21]}$. However, most of them have not proposed an effective method to prevent system blackout in the case of cascading outages.

From the above discussion it is concluded that a special protection scheme is helpful to preserve the integrity of islands following large disturbances in power systems. The special protection scheme may be a kind of adaptive UFLS system, especially designed for this purpose.

In order to detect large imbalances several methods may be used by under frequency relays. In this work two methods are proposed and analyzed:

- Using rate of change of frequency.

- Using the state of tie line circuit breakers.

Rate of change of frequency may be a suitable parameter to enhance the adaptability of underfrequency relays ${ }^{[18-21]}$. This method could be implemented in different ways, some of which are proposed and tested on an actual network in this paper. Using this load shedding method, faster reactions could be obtained for major system failures. This way, system blackouts could be better controlled.

In order to verify ability of the proposed methods, Iranian interconnected grid is simulated appropriately. Khorasan, the greatest province of Iran, is considered to be connected to the grid through a double circuit $400 \mathrm{kV}$ tie line. The Iranian network is an interconnected grid with about $500 \mathrm{HV}$ buses and $30000 \mathrm{MW}$ generation. Khorasan HV network is a 72 bus system with about $2500 \mathrm{MW}$ generation.

The power system under study is modeled as accurately as possible in this work. Distributed and dynamic model is used in this study. Generators, transmission lines, power transformers, Automatic Voltage Regulators (AVR), governors, tap changers, loads and under frequency relays are accurately modeled. A suitable special protection scheme for this double area power system is proposed in the paper and its performance is evaluated.

\section{SPECIAL PROTECTION SCHEMES}

The following definitions are presented in the literature for special protection schemes or system protection schemes:

- System protection schemes are protection strategies designed to detect a particular system condition that is known to cause unusual stress to the power system and to take some kind of predetermined action to counteract the observed condition in a controlled manner ${ }^{[11,23]}$.

- A system protection scheme is designed to detect abnormal system conditions and take predetermined corrective action, other than the isolation of faulted element, to preserve system integrity and provide acceptable system performance ${ }^{[11]}$.

The most common types of system protection schemes are introduced as follows ${ }^{[23]}$ :

- Generator rejection

- Load rejection

- Under frequency load shedding

- System separation

- Turbine valve control

- Load and generator rejection

- Stabilizers

- HVDC controls

- Out of step relaying

- Discrete excitation control

- Dynamic braking

- Generator runback

- VAR compensation

The main objectives of using system protection schemes are listed below ${ }^{[11]}$ :

- Operation of power systems closer to their limits.

- Increase power system security particularly during extreme contingencies.

- Improve power system operation.

- Compensate for the delays in construction programs to extend power system infrastructure.

System protection schemes are usually classified in the following two categories ${ }^{[24]}$.

Response based system protection schemes: In this type of system protection schemes response of the system to disturbances are used in decision making. 
Input signals of the system in this method may be voltage, current, frequency or any other signal of the system. Under frequency load shedding and under voltage load shedding schemes are popular methods in this class.

Event based system protection schemes: In this method decision is based on the state of specific elements in the system such as important transmission lines or generators. This method usually requires a communication link to transmit the state of important elements to the under frequency relays.

In this work UFLS schemes are considered which are usually response-based. However, in some situations they are combined with event based schemes to achieve more effective methods.

\section{CONVENTIONAL UFLS SCHEME}

Conventional UFLS schemes are designed to prevent hazardous frequency decline following the disturbances which may cause considerable mismatches between generation and consumption. Excessive frequency decline may cause damage to the equipments of power system especially turbine blades in power plants. Therefore, every power plant is equipped with a type of under frequency relay which allows each level of frequency decline to exist just for a certain amount of time. After that time if the frequency is not recovered to the permitted level the generator will be tripped. A typical setting for under frequency relays of generators in $60 \mathrm{~Hz}$ system is listed in Table $1^{[25]}$.

Table 1: Generator Under Frequency Relays Setting

\begin{tabular}{ll}
\hline $\begin{array}{l}\text { Under frequency } \\
\text { limit }(\mathrm{Hz})\end{array}$ & \multicolumn{1}{c}{ Maximum time } \\
\hline $60-59.5$ & $\begin{array}{l}\text { Continuous operating } \\
\text { range }\end{array}$ \\
$59.4-58.5$ & 3 minutes \\
$58.4-57.9$ & 30 seconds \\
$57.8-57.4$ & 7.5 seconds \\
$57.3-56.9$ & 45 cycles \\
$56.8-56.5$ & 7.2 cycles \\
Less than 56.4 & Instantaneous trip \\
\hline
\end{tabular}

When frequency of the system falls below under frequency setting of a generator relay, the related generator is tripped. Tripping of this generator intensifies the imbalance between generation and consumption. Therefore, frequency decline will be aggravated and this may cause the under frequency relays of other generators to trip. Consequently a cascading outage of generators is started and eventually the whole system encounters a wide spread blackout.
A conventional approach to prevent these cascading outages is under frequency load shedding. In this method whenever frequency of the system falls below a predetermined value a portion of system load will be dropped.

Design of conventional UFLS schemes involves four stages as explained below.

Determination of the anticipated overload: The anticipated overload determines the amount of protection that is to be provided. This value is obtained from (1). [14]

$\mathrm{L}=\frac{\text { TotalLoad }- \text { TotalGeneration }}{\text { TotalGeneration }}$

where $\mathrm{L}$ is the anticipated overload.

According to (1), the loss of 33 percent of total generation results in 50 percent overload and the loss of 50 percent of total generation results in 100 percent overload. Selection of L is arbitrary, however values greater than 50 percent are not recommended due to possible overshedding during small disturbances.

Determination of the number of load shedding steps:All of the required load to be disconnected may be shed simultaneously, however this approach may lead to overshedding for small disturbances. A suitable alternative may be to divide the load to be dropped among several steps and shed a portion in each step. It seems that incorporating more steps results in more accurate load shedding. However, this is not necessarily true and it must be verified by dynamic studies. Furthermore, increasing the number of steps may cause problems in coordination between successive steps [25]. Typically 3 to 6 steps are recommended [14].

Determination of the amount of load to be shed:The first step is to calculate the total amount of load to be shed to maintain frequency above minimum permissible frequency for the maximum anticipated overload. Total amount of load to be shed is calculated using (2) ${ }^{[14]}$.

$\mathrm{LD}=\frac{\frac{\mathrm{L}}{1+\mathrm{L}}-\mathrm{d}\left(1-\frac{\mathrm{f}}{\mathrm{f}_{\mathrm{n}}}\right)}{1-\mathrm{d}\left(1-\frac{\mathrm{f}}{\mathrm{f}_{\mathrm{n}}}\right)}$

where, $\mathrm{LD}=$ total load that must be shed

$\mathrm{L}=$ per unit overload

$\mathrm{f}=$ minimum permissible frequency

$\mathrm{d}=$ load reduction factor

$\mathrm{f}_{\mathrm{n}}=$ nominal frequency 
Afterward, the amount of load to be shed must be divided among the load shedding steps. Division of load among load shedding steps may be implemented in various ways. If a great amount of load were dropped in the first step, the scheme would be suitable for large mismatches between generation and load. However, it may result in overshedding for small disturbances. Conversely, if a small amount of load is dropped in the first step and a large amount is dropped in the last step, the scheme will be more suitable for small disturbances. However, it may result in large frequency decline for large disturbances. A suitable alternative may be to shed small amount of load in the first step, large amounts in intermediate steps and a small amount in the last step.

Calculating relay settings: The last step is to determine relay settings. After determining the amount of load to be shed in each step, the frequency at which load is dropped for step number one is calculated using relay characteristics curves. Then, setting of the next step is determined using (3).

Setting = previous clearing frequency - safety margin (3) Safety margin is involved to compensate for the inaccuracies of relays, breakers and other uncertainties.

\section{ADAPTIVE UFLS SCHEME}

Conventional UFLS schemes are normally designed for $\mathrm{L}=0.5$ in (1) and (2). Using values greater than 0.5 for parameter $\mathrm{L}$ in the design will result in overshedding for small disturbances. Therefore, in the case of a double area power system with large amount of power transfer through tie lines if, for any reason, the tie lines are disconnected the area which was importing power will be at high risk of blackout. In this case a special protection scheme is required to prevent blackout. An adaptive UFLS scheme could be a good solution.

Several adaptive UFLS schemes are introduced in this section and their performance is analyzed for the special case of this work.

f-df/dt characteristics: In this method frequency settings of the UFLS relays are a function of $\mathrm{df} / \mathrm{dt}$ variable $^{[18]}$. For large values of $\mid \mathrm{df} / \mathrm{dt}$ l higher frequency settings are used, while for small values of ldf/dtl lower frequency settings are used. This way for large imbalances between generation and consumption UFLS relays will quickly be initiated. This may result in higher minimum frequency of the system after the disturbance.

One type of f-df/dt characteristics are depicted in Fig. 1. In this characteristic the region wherein $|\mathrm{df} / \mathrm{dt}|$ is greater than 1.4 is designated large $\mathrm{df} / \mathrm{dt}$ region; the region wherein $|\mathrm{df} / \mathrm{dt}|$ is between 0.9 and 1.4 is designated medium $\mathrm{df} / \mathrm{dt}$ region and the region with Idf/dt। smaller than 0.9 is designated small df/dt region.

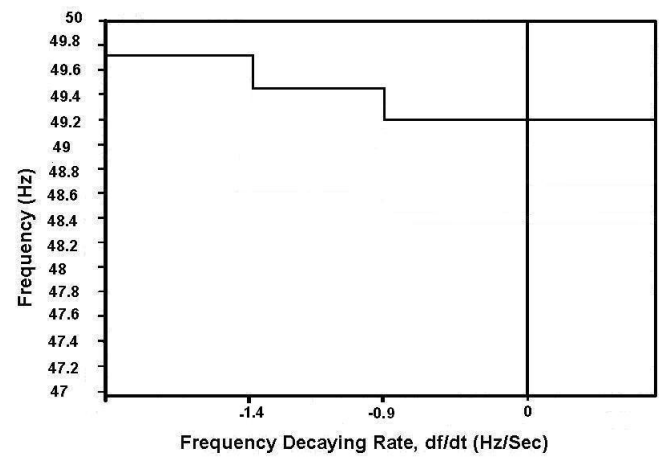

Fig. 1: f-df/dt step characteristic.

$\mathbf{T}_{\mathrm{D}^{-}}$-df/dt characteristics: The basic idea of this method is introduced $\mathrm{in}^{[26]}$. This method is similar to the first method; however instead of using $\mathrm{f}$-df/dt characteristics $\mathrm{T}_{\mathrm{D}}$-df/dt characteristics are used. $\mathrm{T}_{\mathrm{D}}$ is the intentional time delay for load shedding. $\mathrm{T}_{\mathrm{D}}$ does not include the intrinsic delays such as operating time of relays and circuit breakers. In this method for large disturbances small time delays are used while for small disturbances conventional time delays are used. One type of $\mathrm{T}_{\mathrm{D}}-\mathrm{df} / \mathrm{dt}$ characteristic is depicted in Fig. 2.

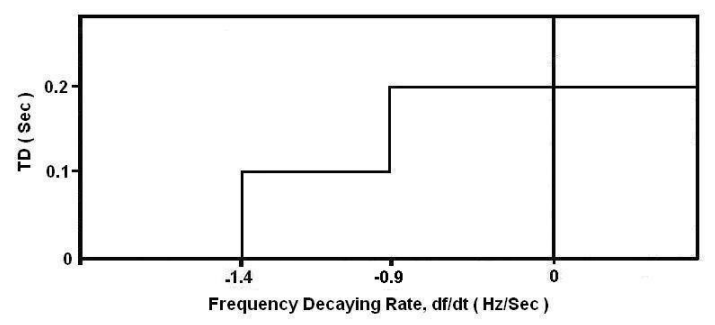

Fig.2: A typical $\mathrm{T}_{\mathrm{D}}$-df/dt characteristic

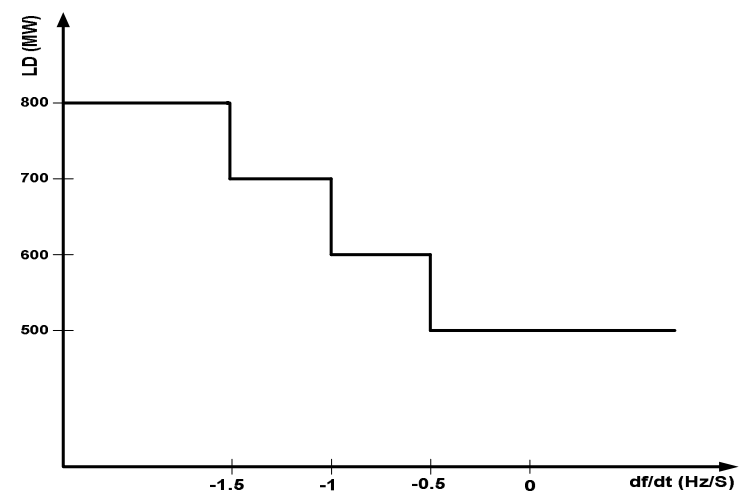

Fig.3: A typical LD-df/dt characteristic 
Adaptive LD-df/dt scheme: In this method the amount of load to be shed is a function of $\mathrm{df} / \mathrm{dt}$ variable. For large disturbances greater amount of load is shed while for small disturbances smaller amount is shed. In this method the UFLS scheme must be designed several times for different levels of $\mathrm{df} / \mathrm{dt}$. The UFLS is designed so that for any disturbance, with its related $\mathrm{df} / \mathrm{dt}$, minimum frequency of the system does not fall below a certain value. This way for each value of $\mathrm{df} / \mathrm{dt}$ a suitable value of $L D$ is calculated where $L D$ is the amount of load to be shed. In other words in this method an LD-df/dt characteristic is used as depicted in Fig. 3.

Event based adaptive UFLS scheme: In the previous methods introduced in sections 4.1 to 4.3 , rate of change of frequency is used to estimate the magnitude of disturbance. These methods are completely response based. In this section an event based method is used to assess the magnitude of disturbance. Outage of important transmission lines or generators may be a good representative of the magnitude of disturbance. For example in a double area power system, outage of tie lines may declare the severity of disturbance for UFLS relays. Sending the state of tie line circuit breakers to UFLS relays may help the relays change their settings according to the disturbance. In this method UFLS relays use a conventional setting by default. If a disturbance causes the tie lines to open, this situation is transmitted to the UFLS relays in order that the relays automatically change their settings to the emergency mode of operation. In the emergency mode one or some of the following strategies may be used:

- Higher frequency settings may be used.

- Smaller time delays may be used.

- Larger amounts of load may be shed.

\section{MODELING A DOUBLE AREA POWER SYSTEM}

In this section a double area power system is modeled for power system analysis. The Iranian network connected to the network of Khorasan province, the greatest province of the country, is modeled for this study. The Iranian network is an interconnected grid with about five hundred $230 \mathrm{kV}$ and $400 \mathrm{kV}$ buses. The amount of generation in this network exceeds 30000 MW. The Khorasan province network is a grid with seventy two $132 \mathrm{kV}$ and $400 \mathrm{kV}$ buses. The amount of generation in this network is about $2300 \mathrm{MW}$. Two networks are connected through a $400 \mathrm{kV}$ tie line.

An accurate modeling is used in this study. A detailed distributed and dynamic model of the system is developed. The conventional lump and static system model is not able to provide accurate results and does not provide access to various system variables at different locations. The distributed and dynamic model of the network involves generators with governors and AVRs, transmission lines, power transformers, tap changers, shunt capacitors, shunt reactors, loads and under frequency relays. Fig. 4 depicts a schematic diagram of the network and Fig. 5 depicts the single line diagram of Khorasan network.

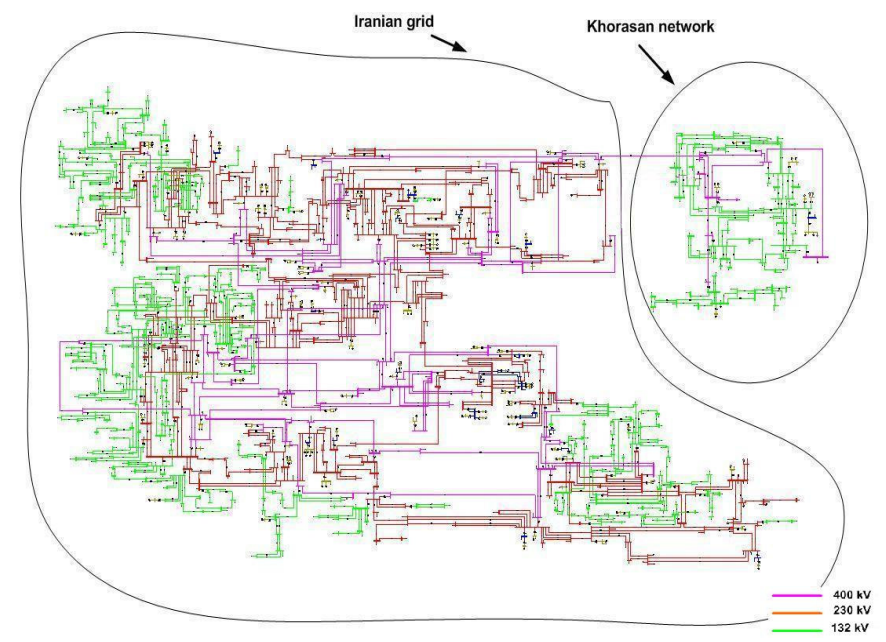

Fig. 4: Schematic diagram of the Iranian network connected to the Khorasan network.

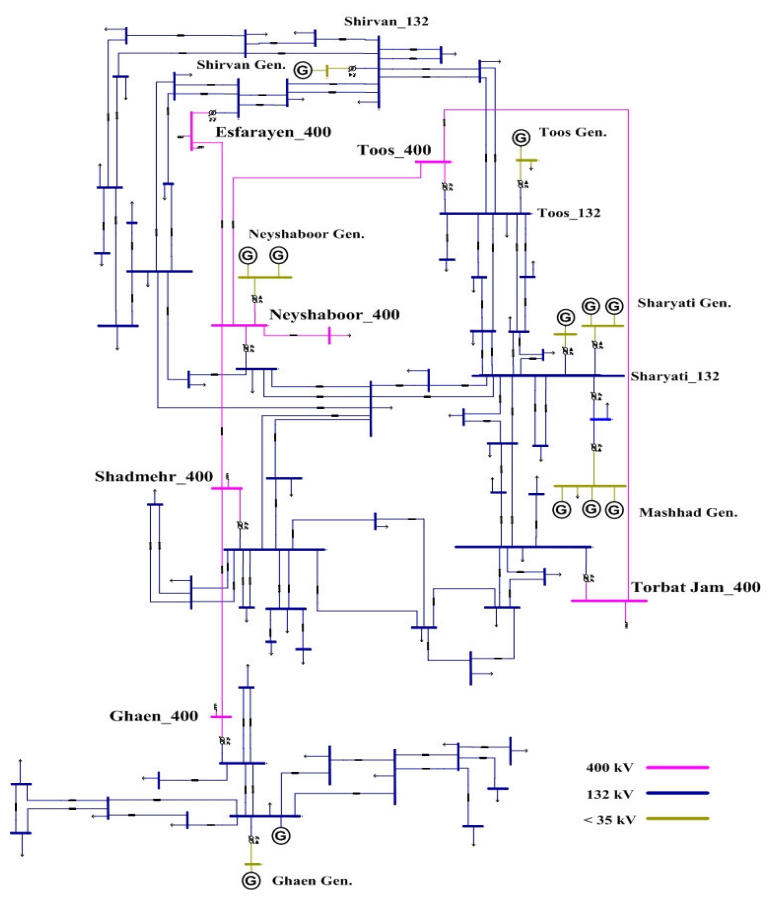

Fig.5: Single line diagram of the Khorasan network. 
In this section modeling of power system components is described in detail:

Generator modeling: The forth order model of the generator is used in this study ${ }^{[27,28]}$. Since damper windings are included in this model oscillations are small and realistic. Using the first order model provides large generator oscillations after system disturbance which are not realistic.

Governor modeling: Governors are modeled according to the simplified model of ${ }^{[27]}$. Since the responses of boiler and reheaters are slow they are not modeled in load shedding studies.

AVR modeling: The IEEE type DC1 model is used for AVR modeling ${ }^{[27,28]}$.

Load modeling: The EPRI static load model is used in this work $^{[29]}$.

\section{DESIGN OF CONVENTIONAL AND ADAPTIVE UFLS SCHEMES}

In this section conventional UFLS scheme and the four adaptive schemes introduced in section 4 are designed for the Khorasan network. The purpose of this study is to evaluate performance of the schemes in preventing blackout in the Khorasan network after isolation of the network from Iranian grid.

Conventional UFLS design: In this subsection conventional UFLS scheme is designed for Khorasan network according to the method previously described. Design is based on the following parameters:

- $\mathrm{L}=0.5$

- Total load = $1774 \mathrm{MW}$

- $\mathrm{d}=1.7$

- $\mathrm{f}($ minimum permissible frequency $)=48 \mathrm{~Hz}$

Using the above data and (2), value of 0.285 per unit or $510 \mathrm{MW}$ is obtained for the parameter LD. The value of 0.2 second time delay for frequency relays and frequency settings according to the Table 2 are used in the simulation. These values are in accordance with the real amounts used for the Khorasan relays.

Table 2: Frequency settings of conventional UFLS scheme

\begin{tabular}{ccc}
\hline $\begin{array}{c}\text { Step } \\
\text { Number }\end{array}$ & $\begin{array}{c}\text { Frequency } \\
\text { setting } \\
(\mathrm{Hz})\end{array}$ & $\begin{array}{c}\text { Amount of load to } \\
\text { be shed in each step } \\
\text { (percentage of LD ) }\end{array}$ \\
\hline 1 & 49.2 & $20 \%$ \\
2 & 49 & $20 \%$ \\
3 & 48.7 & $30 \%$ \\
4 & 48.4 & $30 \%$ \\
\hline
\end{tabular}

The following factors are considered in choosing the loads to be shed.

- The loads to be shed are chosen among loads with low degree of importance.

- The loads to be shed are chosen in a way that they are distributed in the whole system. In other words they are not concentrated in any specific area.

Adaptive UFLS with f-df/dt characteristic: Design of UFLS scheme in this method is described in $^{[2]}$. A summary is presented in this section. The design involves 5 stages.

Distributed and dynamic modeling of the system: In the first stage, distributed and dynamic model of the power system under study is simulated.

Simulation of several outage scenarios: In the second stage several generation and tie line outage scenarios, with different values of anticipated overload are simulated on the system. In this stage under frequency relays are disabled.

f-df/dt loci of the system: f-df/dt loci of the slack busbar, for all scenarios of stage 2.2 are obtained from the simulation results. For example the locus for a large disturbance with $720 \mathrm{MW}$ generation outage is depicted in Fig. 6.

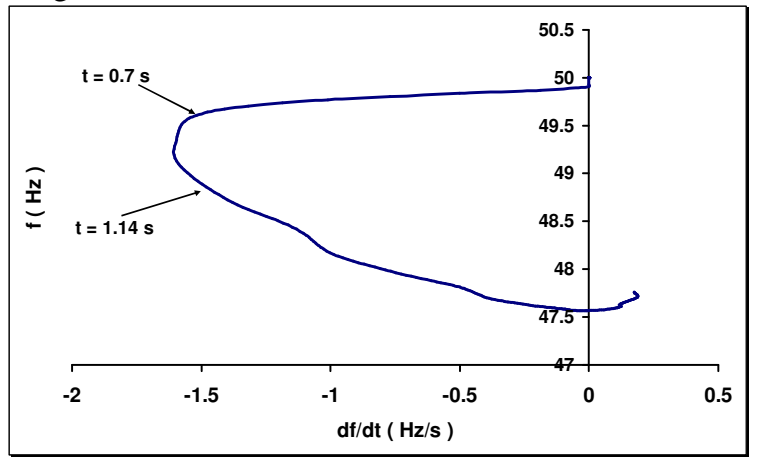

Fig. 6: Locus of $\mathrm{f}-\mathrm{df} / \mathrm{dt}$ for $720 \mathrm{MW}$ generation outage in Khorasan network.

Setting the adaptive UFLS system: Using the loci obtained in stage 2.3, the first step of under frequency relays, characteristics of which were depicted in Fig. 1, are determined. The parameters are determined such that:

- For large disturbances under frequency relays operate in large $\mathrm{df} / \mathrm{dt}$ region of the characteristics shown in Fig. 1. 
- For medium disturbances the relays operate in medium df/dt region.

- For small disturbances the relays operate in small df/dt region.

Testing the design: After setting parameters, the design must be tested on the model of power system for all scenarios of stage 2.2 in different operating conditions. In this stage it may be necessary to modify parameters.

Adaptive UFLS with $\mathbf{T}_{D^{-}}$df/dt characteristic: In the first stage of design, similar to the previous method, distributed and dynamic model of power system under study is simulated. In the second stage several generation outage scenarios are simulated on the systems under study. Initially in this stage under frequency relays are disabled. The left side of the curve in Fig. 6, e.g. from $t=0.7 \mathrm{~s}$ to $\mathrm{t}=1.14 \mathrm{~s}$, is important in the design. Values of df/dt setting which should be used in Fig. 2 are determined from the values of $\mathrm{df} / \mathrm{dt}$ in the left side of f-df/dt loci. For example if for a large disturbances $\mathrm{df} / \mathrm{dt}$ in the left side of $\mathrm{f}-\mathrm{df} / \mathrm{dt}$ locus is around $-1.5 \mathrm{~Hz} / \mathrm{s}$, the minimum $\mathrm{df} / \mathrm{dt}$ setting in $\mathrm{T}_{\mathrm{D}}-\mathrm{df} / \mathrm{dt}$ characteristics is chosen $-1.4 \mathrm{~Hz} / \mathrm{s}$ as depicted in Fig. 1. The safety margin of $0.1 \mathrm{~Hz} / \mathrm{s}$ is included to compensate for the following uncertainties:

- Differences between f-df/dt characteristics of slack busbar and load busbars where $\mathrm{df} / \mathrm{dt}$ is calculated.

- Variations of f-df/dt characteristics in different operational conditions of power system.

The other df/dt setting point in Fig. 2 belongs to medium disturbances and it is calculated similar to the previously discussed case. For Khorasan network it is equal to $-0.9 \mathrm{~Hz} / \mathrm{s}$ as depicted in Fig. 2.

Adaptive UFLS with LD-df/dt characteristic: In this section an adaptive UFLS scheme, using LD-df/dt characteristic, is designed for Khorasan network. In this method the amount of load to be shed is a function of $\mathrm{df} / \mathrm{dt}$. The characteristic used in this work is depicted in Fig. 7. The characteristic contains three separate regions. The large $\mathrm{df} / \mathrm{dt}$ region is related to a major disturbance, e.g. the islanding condition in which the tie line is tripped due to a disturbance. The medium $\mathrm{df} / \mathrm{dt}$ region is related to medium disturbances and the small $\mathrm{df} / \mathrm{dt}$ region is related to the small disturbances. The values of DF1 and DF2 in which the transition between separate regions occur are obtained from the dynamic studies of the system similar to the calculations of previous section. This way the values of -1.4 and -0.9 $\mathrm{Hz} / \mathrm{s}$ are obtained for DF1 and DF2 respectively.

The next step is to determine the values of LD1, LD2 and LD3. LD1 is related to small disturbances. In this work small disturbances are those with the percentage of generation outage less than $20 \%$. Therefore according to (1) $\mathrm{L}$ is equal to 0.25 in this region. Using (2) and value of 0.25 for $L$, the value of 0.14 P.U. or $250 \mathrm{MW}$ is obtained for LD1. Other parameters of (2) are equal to those of conventional method.

LD2 is related to medium disturbances. In this work medium disturbances are those with the percentage of generation outage between 20 and $33 \%$. Therefore according to (1) $\mathrm{L}$ is equal to 0.5 in this region. Using (2) and value of 0.5 for $L$, the value of 0.285 P.U. or $510 \mathrm{MW}$ is obtained for LD2.

LD3 is related to large disturbances including large generation outages or tie line outage. In this work large disturbances are those with the percentage of generation outage greater $33 \%$. In this region the value of $\mathrm{L}$ is supposed to be 1.32 which is related to the tie line outage which produces the largest mismatch of generation and load. In this design it is supposed that the amount of power transfer between two areas is 1100 MW, based on the contract between two companies. Obviously the deign may be revised and adapted for any other agreement between the companies. Using the amounts of $1100 \mathrm{MW}$ power transfer between the areas, $1774 \mathrm{MW}$ for total load, $764 \mathrm{MW}$ for total generation of Khorasan network and $90 \mathrm{MW}$ for losses in Khorasan network the value of 1.32 is obtained for $\mathrm{L}$ according to (1). Using this value for $\mathrm{L}$ and the other parameters equal to that of conventional method, the amount of 0.537 per unit or $950 \mathrm{MW}$ is obtained for LD3.

It must be considered that the actual amount of power transfer through tie line is about $300 \mathrm{MW}$ from Khorasan to the Iranian grid. However in this work in order to model a small network that is largely dependent on another network the power transfer is accepted to be about $1100 \mathrm{MW}$ from Iran to Khorasan network.

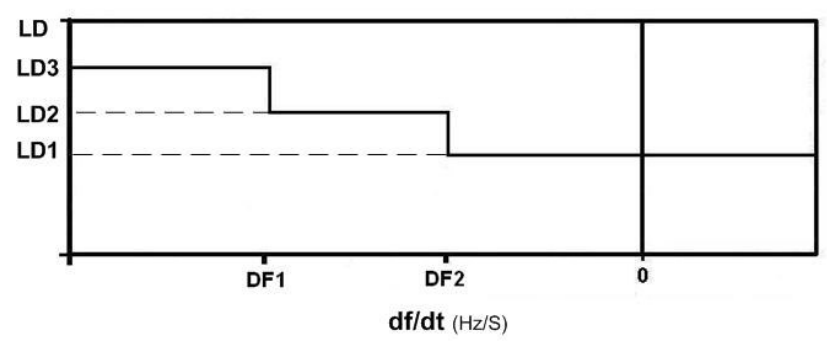

Fig.7: LD-df/dt characteristic 
Event based adaptive UFLS scheme: In this section an event based adaptive UFLS scheme is designed for Khorasan network, connected to the Iranian grid. In this method instead of using $\mathrm{df} / \mathrm{dt}$ to recognize the outage of the tie line, a communication link is used. Whenever the tie line is tripped due to a disturbance, a signal is transmitted to the UFLS relays. Receiving this signal causes the UFLS relays to change their settings and transfer to the emergency mode of operation. In this study outage of tie line causes the UFLS system to shed larger amount of load compared with the conventional mode. Therefore, in order to implement this method some specific relays must exist in the system which will be activated only if the outage signal of tie line is received. This way in the event of tie line outage a large amount of load is shed from the system. In this method, two different modes are designed for the UFLS system. The normal mode is the conventional one which is designed for $\mathrm{L}=0.5$. In this case the value of $510 \mathrm{MW}$ is obtained for LD, according to (2).

The emergency mode is related to the outage of tie line. For each value of power transfer between two areas, the UFLS scheme may be adapted to that situation. In this study the power transfer of $1100 \mathrm{MW}$ is used for the design. It may be necessary to revise the design in different seasons. Using the amount of $1100 \mathrm{MW}$ power transfer, the value of $950 \mathrm{MW}$ is obtained for $\mathrm{LD}$, according to (2). In previous section it was completely described how to obtain the values of 510 and $950 \mathrm{MW}$ for LD. A delay equal to 5 milliseconds is also considered for the operation and delays of communication facilities.

\section{SIMULATION RESULTS}

The UFLS schemes, designed in section 6 , are modeled appropriately. At the first stage no UFLS relays are used. In this case several tie line outages are simulated on the system with different values of power transfer before the outage. The amount of power transfer is increased until the system becomes unstable after the tie line outage and cascading outage of generators occurs. Maximum possible power transfer through the tie line so that the system would stay stable after the outage of tie line is $640 \mathrm{MW}$ in the case no load shedding is used. System reserve is enough to compensate for such a great disturbance.

The same scenario is performed for the system with conventional and adaptive UFLS schemes. In each case maximum possible amount of power transfer, so that the system would be stable after the tie line outage, is obtained. The results are listed in Table 3. Table 3 implies that without any UFLS scheme maximum 640 MW power transfer through tie line is permitted so that if the tie line is tripped the system will remain stable. For the case of conventional UFLS scheme this limit is $1100 \mathrm{MW}$ i.e. if $1100 \mathrm{MW}$ power or less is transferred through tie line and it is tripped the system will remain stable. This limit is $1030 \mathrm{MW}$ and $1040 \mathrm{MW}$ for adaptive load shedding with $\mathrm{T}_{\mathrm{D}^{-}} \mathrm{df} / \mathrm{dt}$ and $\mathrm{f}-\mathrm{df} / \mathrm{dt}$ characteristics, respectively.

In the case of adaptive load shedding with LD-df/dt characteristic and event-based adaptive load shedding there is no limit for power transfer through tie line from frequency stability point of view. It means that for any value of power transfer through tie line, the balance of generation and consumption may be recovered by these methods. In these cases transient stability and dynamic stability restrictions limit the amount of power transfer through tie line.

Table 3: Maximum permissible amount of power transfer

\begin{tabular}{|c|c|}
\hline Method & $\begin{array}{l}\text { Maximum permissible amount } \\
\text { of power transfer (MW) }\end{array}$ \\
\hline $\begin{array}{l}\text { Without load } \\
\text { shedding }\end{array}$ & 640 \\
\hline $\begin{array}{l}\text { Conventional } \\
\text { UFLS }\end{array}$ & 1010 \\
\hline $\begin{array}{l}\text { Adaptive UFLS } \\
\text { with } \mathrm{T}_{\mathrm{D}} \text {-df/dt } \\
\text { characteristic }\end{array}$ & 1030 \\
\hline $\begin{array}{l}\text { Adaptive UFLS } \\
\text { with } \mathrm{f}-\mathrm{df} / \mathrm{dt} \\
\text { characteristic }\end{array}$ & 1040 \\
\hline $\begin{array}{l}\text { Adaptive UFLS } \\
\text { with LD-df/dt } \\
\text { characteristic }\end{array}$ & - \\
\hline $\begin{array}{l}\text { Event based } \\
\text { adaptive UFLS }\end{array}$ & - \\
\hline
\end{tabular}

Fig. 8 shows the frequency of some busbars in Khorasan network for the disturbance of tie line outage in the case of using conventional UFLS. The amount of power transfer between areas before the tie line outage is $1100 \mathrm{MW}$. In this case, although all four steps of conventional UFLS scheme operate and 510 MW load is shed, the system does not remain stable and frequency does not recover.

Fig.9 shows the frequency of some busbars in Khorasan network for the same disturbance as Fig. 8 in the case of adaptive UFLS with LD-df/dt characteristic. In this case the system remains stable and frequency begins to increase after about 1.5 seconds. In this scheme 950 MW load is shed instead of $510 \mathrm{MW}$ of conventional scheme and the system is preserved from instability. 


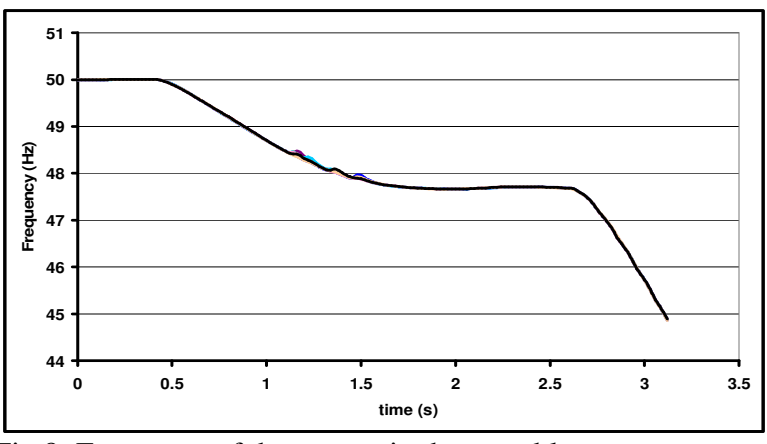

Fig.8: Frequency of the system in the unstable case

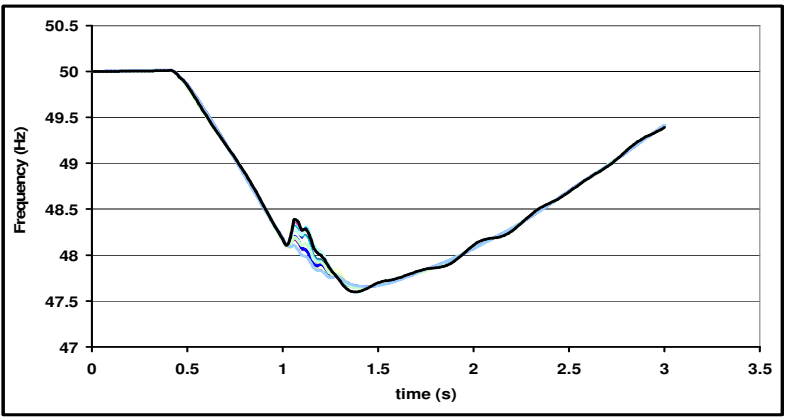

Fig.9: Frequency of the system in the stable case

In order to further illustrate the performance of different UFLS schemes, several tie line outages are simulated on the system with different values of power transfer through tie line before the outage. For each value of generation outage, frequency of the slack busbar for different UFLS schemes is obtained by simulation. Figs. 10 to 12 depict the frequency of slack busbar for the tie line outages with 700, 900 and $1100 \mathrm{MW}$ power transfer before the outage, respectively.

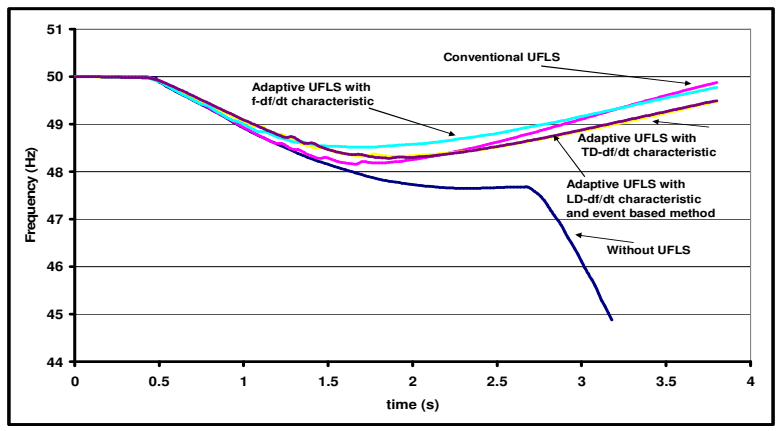

Fig. 10: Frequency of the slack busbar for the tie line outage with $700 \mathrm{MW}$ power transfer

It is concluded from Fig. 10 that if the amount of power transfer through tie line is $700 \mathrm{MW}$ and the tie line is tripped due to any disturbance, the system without
UFLS scheme does not stay stable and cascading outage of generators due to frequency decline causes blackout in the Khorasan network. This simulation reveals the necessity of UFLS scheme in a double area power system when the amount of power transfer between areas is relatively large. Performance of UFLS schemes is not so much different in this case. However, the adaptive scheme with $\mathrm{f}-\mathrm{df} / \mathrm{dt}$ characteristic results in a higher minimum frequency, compared with other load shedding methods. In all UFLS schemes designed in this work, all four steps of under frequency relays have operated for this outage.

Since the system will remain stable after the tie line outage, all UFLS shedding schemes designed in this work could be considered acceptable for this outage case.

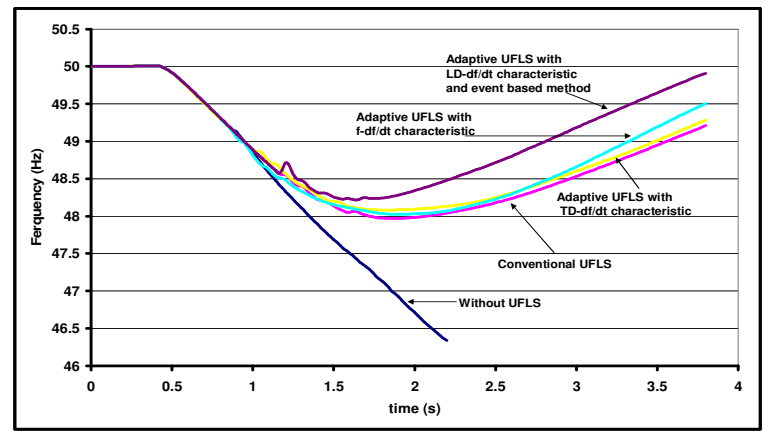

Fig. 11: Frequency of the slack busbar for the tie line outage with $900 \mathrm{MW}$ power transfer

Fig .11 is related to a greater disturbance, compared with Fig. 14. In this case $900 \mathrm{MW}$ power is transferred from the Iran grid to the Khorasan network before the disturbance. Again the system will become unstable without UFLS system. In this case the results of conventional UFLS scheme, adaptive scheme with f$\mathrm{df} / \mathrm{dt}$ characteristics and adaptive scheme with $\mathrm{T}_{\mathrm{D}}$-df/dt characteristic are almost the same while the results of adaptive scheme with LD-df/dt characteristic and eventbased adaptive UFLS scheme are more acceptable since their minimum frequencies are approximately $0.5 \mathrm{~Hz}$ greater than other schemes. All four steps of under frequency relays operate for this disturbance.

Fig .12 is related to an extremely large disturbance. In this case 1100 MW power is transferred to the Khorasan network.

After the disturbance, in addition to the system without UFLS scheme, the systems with conventional UFLS scheme, adaptive scheme with $\mathrm{T}_{\mathrm{D}}$-df/dt characteristic and adaptive scheme with f-df/dt characteristic will all become unstable, while the systems with event based adaptive scheme and adaptive scheme with LD-df/dt characteristic will remain stable. 


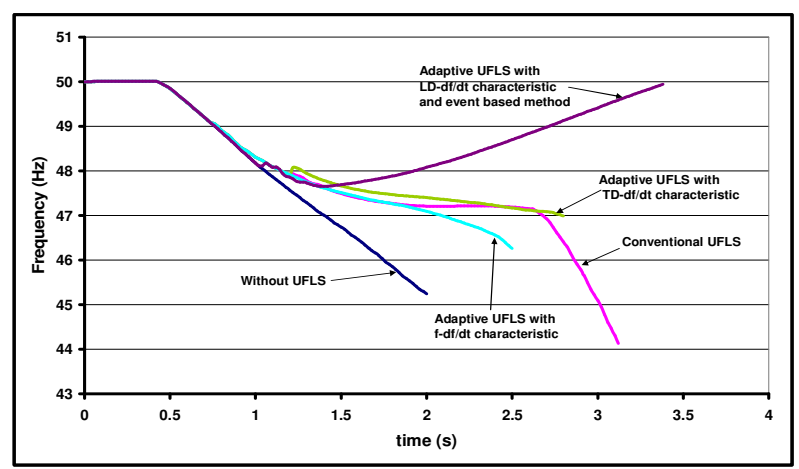

Fig. 12: Frequency of the slack busbar for the tie line outage with $1100 \mathrm{MW}$ power transfer

In the latter methods for each value of power transfer through the tie line, the UFLS scheme is completely adapted to that situation. Therefore, if a tie line outage is detected by the UFLS system, the system will go the emergency mode of operation and the amount of load to be shed is increased, compared with ordinary generation outage scenarios. The results of Table 3 confirm this fact.

The following points are the important conclusions obtained through the simulations:

- Equipping the system with any type of UFLS system considerably increases the amount of permissible power transfer through the tie line.

- Performance of the three methods of conventional UFLS, adaptive UFLS with f$\mathrm{df} / \mathrm{dt}$ characteristic and adaptive UFLS with $\mathrm{T}_{\mathrm{D}^{-}}$ $\mathrm{df} / \mathrm{dt}$ characteristic is not so much different in this system.

- Adaptive UFLS with LD-df/dt characteristic and event based adaptive UFLS schemes are completely acceptable in this case. These two schemes may be designed such that for any scheduled power transfer through tie line outage of tie line would not result in cascading outage of generators.

- Adaptive UFLS with LD-df/dt characteristic has the advantage that it does not require communication link. While event based adaptive UFLS scheme does require a reliable communication link.

- Event based adaptive UFLS scheme has the advantage that recognition of tie line outage is more precise in this method, compared with response based schemes. In response based schemes design of the adaptive relays using $\mathrm{df} / \mathrm{dt}$ could become somewhat complicated, since $\mathrm{df} / \mathrm{dt}$ at different buses of the system are not completely the same.
- Event based adaptive UFLS scheme has the advantage that using this method it is not required to calculate $\mathrm{df} / \mathrm{dt}$. Accurate calculation of $\mathrm{df} / \mathrm{dt}$, especially during disturbances, may be difficult and it requires advanced filtering techniques.

\section{CONCLUSIONS}

In this paper several UFLS schemes were introduced and they were used as special protection schemes to prevent blackout in a double area power system following a disturbance causing the tie line between two areas to open. Complete methods to design the UFLS schemes were also presented in detail. After completing the design it was tested by simulation. Several stable and unstable cases were simulated for each scheme. Results of simulations confirm the necessity of using a UFLS scheme in the case of double area power system. Among different UFLS schemes analyzed in this paper, conventional UFLS scheme, adaptive UFLS scheme with $\mathrm{f}-\mathrm{df} / \mathrm{dt}$ characteristic and adaptive UFLS with $\mathrm{T}_{\mathrm{D}^{-}}$-df/dt characteristic are suitable methods; however they have some restrictions in the amount of high power transfer through the tie line.

Adaptive UFLS scheme with LD-df/dt characteristic and event based adaptive UFLS scheme are complete methods and they protect the special system against blackout after tie line outage, almost with no restriction. The adaptive UFLS scheme with LD-df/dt characteristic has the advantage of protecting the system without any communication link. However, it suffers from the disadvantage of using $\mathrm{df} / \mathrm{dt}$, calculation of which might be complicated. The event based adaptive UFLS scheme requires communication link instead of calculating $\mathrm{df} / \mathrm{dt}$. Therefore, the design is rather simple and more accurate; however it may be more expensive, compared with response based schemes. As shown in the paper, for a double area power system if the amount of power transfer exceeds a certain value, UFLS scheme is necessary. Increasing the amount of power transfer above a threshold necessitates the use of either adaptive scheme with LD$\mathrm{df} / \mathrm{dt}$ characteristic or event based adaptive scheme.

\section{REFERENCES}

1. S. H. Horowitz, A. G. Phadke, 'Boosting immunity to blackouts', IEEE Power and Energy Magazine, Sept./Oct. 2003

2. H. Seyedi, M. Sanaye-Pasand, M. R. Dadashzadeh, Design and simulation of an adaptive load shedding algorithm using a real network, IEEE Power India Conference, April 10-12, 2006 
3. S. Tamronglak, S. H. Horowitz, A. G. Phadke, J. S. Thorp, Anatomy of power system blackouts: preventive relaying strategies, IEEE Trans. on Power Delivery, vol. 11, No. 2, April 1996

4. C. Vournas, Technical summary on the Athens and southern Greece blackout of July 12, 2004, National Technical University of Athens Report

5. S. Corsi, C. Sabelli, General blackout in Italy, Sunday September 28, 2003, h. 03:28:00, IEEE Power Engineering Society General Meeting, 2004, Vol. 2, Pages 1691-1702

6. Power failure in eastern Denmark and southern Sweden on 23 September 2003, Final report on the course of events, Elkraft System, November 2003

7. Technical analysis of the August 14, 2003 blackout, what happened, why and what did we learn?, North American Electric Reliability Council, Final Report, July 23, 2004

8. C. W. Taylor, D. C. Erickson, Recording and analyzing the July 2 cascading outage, IEEE Computer Applications in Power, Vol. 10, Jan. 1997, Pages 26-30

9. M. Thaden, Analysis of a major load island outage on the Potomac electric power company system, IEEE Trans. on Power Systems, vol. 14, No. 1, Feb. 1999

10. D. N. Kosterev, C. W. Taylor, W. A. Mittelstadt, Model validation for the August 10, 1996 WSCC system outage, IEEE Trans. on Power Systems, vol. 14, No. 3, Aug. 1999

11. M. Jonson, Protection strategies to mitigate major power system breakdowns, Ph.D. Dissertation, Chalmers University of Technology, Goteborg, Sweden, 2003

12. C. W. Taylor, Concepts of undervoltage load shedding for voltage stability, IEEE Trans. on Power Delivery, vol. 7, No. 2, April. 1992

13. P. M. Anderson, M. Mirheydar, An adaptive method for setting underfrequency load shedding relays, IEEE Trans. on Power Systems, vol. 7, No. 2, May 1992

14. J. R. Jones, W. D. Kirkland, Computer algorithm for selection of frequency relays for load shedding, Computer Applications in Power, Vol. 1, Jan. 1988, Pages 21-25

15. M. Etezadi-Amoli, On underfrequency load shedding schemes, Proceedings of the 22'nd Annual North American Power Symposium, Oct. 1990, Pages 172-180

16. B. Delfino, S. Massucco, A. Morini, P. Scalera, F. Silvestro, Implementation and comparison of different underfrequency load shedding schemes, IEEE Power Engineering Society Summer Meeting, July 2001, Vol. 1, Pages 307-312
17. C. Concordia, L. H. Fink, G. Poullikkas, Load shedding on an isolated system, IEEE Trans. on Power Systems, vol. 10, No. 3, Aug. 1995

18. L. J. Shih, W. J. Lee, J. C. Gu, Y. H. Moon, Application of $\mathrm{df} / \mathrm{dt}$ in power system protection and its implementation in microcontroller based intelligent load shedding relay, Industrial and Commercial Power Systems Technical Conf., May 1991, Pages 11-17

19. J. Jung, C. C. Liu, S. L. Tanimoto and V. Vittal, Adaptation in load shedding under vulnerable operating conditions, IEEE Trans. on Power Systems, vol. 17, No. 4, Nov. 2002

20. T. Tomsic, G. Verbic and F. Gubina, Revision of the underfrequency load shedding scheme of the Slovenian power system, IEEE PES General Meeting, 2005

21. D. L. H. Aik, A general-order system frequency response model incorporating load shedding: analytic modeling and application, IEEE Trans. on Power Systems, vol. 21, No. 2, May 2006

22. P. Cote, S. P. Cote, M. Lacroix, Programmable load shedding systems, Hydro Quebec experience, IEEE Power Engineering Society Summer Meeting, July 2001, Vol. 2, Pages 818-823

23. P. M. Anderson, B. K. Le Reverend, Industry experience with special protection schemes, IEEE Trans. on Power Systems, vol. 11, No. 3, Aug. 1996

24. C. Moors, D. Lefebvre, T. Van Custem, Design of load shedding against voltage instability, IEEE Power Engineering Society Winter Meeting, Jan. 2000, Vol. 2, Pages 1495-1500

25. WECC coordinated off-nominal frequency load shedding and restoration plan, Final report, Nov. 1997

26. M. F. Abusharkh, A. A. Hiyasat, Load shedding scheme of the Jordanian national power system, 4'th International Conf. on Developments in Power System Protection, April 1989, Pages 96-101

27. PSAF software user's guide and reference manual, CYMSTAB for windows, CYME international inc., Sept. 1999

28. P. M. Anderson, A. A. Fouad, Power system control and stability, IEEE press, 2003

29. P. S. Kundur, Power system stability and control, Mc Grow-Hill, Inc. 1993 